\title{
Prüfwesen wird generalüberholt - steigt 2017 die Regressgefahr?
}

\begin{abstract}
_ Die Wirtschaftlichkeitsprüfungen werden komplett neu strukturiert - so hat es der Gesetzgeber im GKV-Versorgungsstärkungsgesetz vorgesehen. Das bedeutet unter anderem, dass ab dem 1 . Januar 2017 die Richtgrößenprüfungen nicht mehr Regelprüfmethode für Arznei- und Heilmittelverordnungen sind. KVen und Kassen müssen nun geeignete Alternativen vereinbaren. Auf Bundesebene gibt es bereits einheitliche Rahmenvorgaben, die nun in den RegionalKVen umgesetzt werden müssen.

$\$ 106 \mathrm{~b}$ Abs. 2 SGB V sieht vor, dass die Prüfungsvereinbarungen insbesondere den Umfang bestimmen müssen, in dem Wirtschaftlichkeitsprüfungen mindestens durchgeführt werden sollen.
\end{abstract}

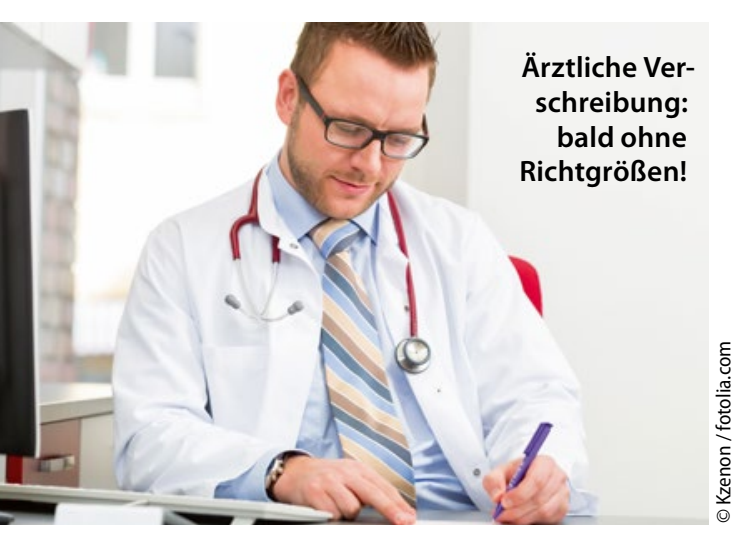

\section{Kassenpatienten ab sofort zur PET überweisen}

—Erweiterung der GKV-Leistungen: Seit dem 1. Januar 2016 sind Überweisungen zur diagnostischen Positronenemissionstomografie (PET) und der diagnostischen PET mit Computertomografie (CT) auch bei Kassenpatienten
Außerdem muss in dem Verfahren sichergestellt sein, dass Ärzte, die bei statistischen Prüfungen erstmalig auffällig werden, zunächst individuell beraten und erst später mit Nachforderungen konfrontiert werden.

Sofern regional statistische Prüfungsmethoden vereinbart werden, sollen vorrangig Auffälligkeitsprüfungen zum Einsatz kommen. Der Prüfzeitraum soll dabei ein Jahr umfassen. Die Zahl der Prüffälle soll maximal 5\% der Ärzte einer Fach- bzw. Vergleichsgruppe betreffen. Neben dieser Höchstquote sollen in den regionalen Vereinbarungen auch Auffälligkeitsgrenzen bei vereinbarten Zielwerten festgelegt werden. Ärzte, die nur wenig verordnen, können von den Prüfungen ausgenommen werden.

\section{MMW-KOMMENTAR}

Es ist wichtig, dass in den Rahmenvorgaben der weiterhin gültigen gesetzlichen Vorschrift Rechnung getragen wurde, dass bei erstmaliger Auffälligkeit bei statistischen Prüfungen zunächst eine individuelle Beratung erfolgen muss, bevor weitere Maßnahmen festgesetzt werden können. Beachtenswert ist, dass dies für jeden Verordnungsbereich gesondert gilt. Die Prüfer können deshalb je nach Prüfungsvereinbarung z. B. Phy- sio- und/oder Ergotherapie, Stimm-, Sprechund Sprachtherapie oder Podologie gesondert betrachten.

$\mathrm{Neu}$ ist, dass eine festgesetzte Maßnahme nach fünf Jahren verjährt. Nach dieser Frist gelten deshalb Ärzte, die erneut ins Visier der Prüfer geraten, wieder als „erstmalig auffällig", haben also erneut Anspruch auf eine Beratung. Bei neu zugelassenen Ärzten kann erst ab dem dritten Prüfzeitraum nach der Zulassung - also nach drei Jahren - eine Beratung festgesetzt werden.

Verordnete Leistungen von Ärzten, die an Anwendungsbeobachtungen nach $\S 67$ Abs. 6 des Arzneimittelgesetzes teilnehmen, sollen bevorzugt geprüft werden. Die KBV wird deshalb den KVen weiterhin die teilnehmenden Ärzte zur Weiterleitung an die Prüfungsstelle mitteilen. Bei Heilmittelverordnung soll künftig die Diagnoseliste der bundesweiten Vereinbarung über Praxisbesonderheiten Bestandteil der Rahmenvorgaben zur Wirtschaftlichkeitsprüfung sein. Um einen besonderen Verordnungsbedarf geltend zu machen, muss weiterhin neben der ICD-10-Diagnose der entsprechende Indikationsschlüssel auf den Verordnungsvordrucken eingetragen werden. Damit dies in der Praxis leichter vonstattengeht, müssen ab 2017 alle Praxisprogramme solche Informationen aus der Arzneimittelrichtlinie beinhalten. möglich - und das auch außerhalb einer stationären Behandlung.

\section{MMW-KOMMENTAR}

Die neuen Leistungen werden vom Vorhandensein einer Qualitätssicherungsvereinba- rung abhängig gemacht. Bis diese in Kraft getreten ist, können die Leistungen ohne entsprechende Genehmigung längstens bis zum 30. Juni 2016 von allen Praxen zum Einsatz gebracht werden, die über ein solches Gerät verfügen. 